\title{
Appearance of a low superheat "quasi-Leidenfrost" regime for boiling on superhydrophobic surfaces
}

\author{
${ }^{a}$ Department of Engineering and Applied Science, University of Bergamo, Viale Marconi 5, 24044 Dalmine, Italy \\ ${ }^{\mathrm{b}}$ Laboratoire de Physique des Surfaces et des Interfaces, Université de Mons, Av. Maistriau 19 B, 7000 Mons, Belgium \\ c Università di Pisa - DESTEC, Largo L. Lazzarino, 1, 56122, Pisa, Italy \\ ${ }^{d}$ University of Brighton, School of Computing, Engineering and Mathematics, Lewes Road, BN2 4GJ, Brighton, UK
}

*Corresponding author. E-mail address: m.marengo@brighton.ac.uk

\begin{abstract}
Pool boiling experiments were performed with degassed water on stainless steel substrates with different surface topographies and wettabilities. Boiling curves and visual observations of the boiling process have been carried out. The onset of nucleate boiling (ONB) has been measured and the influence of roughness and wettability has been quantified. Boiling curve shape is different between the hydrophilic and the superhydrophobic case, superhydrophobic surfaces reaching the ONB heat flux at lower superheat and presenting a quasi-Leidenfrost regime, without showing the typical boiling curve. Bubbles are easier to form on superhydrophobic surfaces, therefore the nucleation temperature is smaller, and bubbles are larger and stable. The ONB appears after less than $5 \mathrm{~K}$ of superheat on superhydrophobic surfaces, while on hydrophilic surfaces, with the same surface roughness, the superheat is above $7 \mathrm{~K}$. Furthermore, superhydrophobic samples with different roughness present the same boiling curve, meaning that, when the contact angle exceeds a certain value, the wettability has a predominant role on the surface roughness.
\end{abstract}

Keywords: wettability, superhydrophobicity, pool boiling, boiling onset, nucleation

\section{Introduction}

Boiling is the rapid vaporization of a liquid and it occurs when a liquid is heated above its boiling point. The vaporization phenomena and the local heat transfer depend mainly upon two surface parameters: the surface topography [1] [2] and the surface wettability [2] [3]. Wettability and roughness are known to play an important role in boiling heat transfer and they are strongly related. Many studies on boiling are performed with changes both in the surface roughness and the topography [4] [5] [6]. Only recently authors have used glass or silicon wafer surfaces with self-assembling monolayer over the substrate in order to modify the wettability of a solid without affecting its topography [7] [8]. The role of surface roughness and surface cavities on nucleation is known since about 1960, even if experimental investigations on roughness effects in pool boiling began systematically about 30 years ago, when enhanced pool boiling with porous coatings received greater attention. Investigations on enhanced boiling from heated surfaces with micro-pin-fin arrays and micro-cavity arrays [9] [10] began in 1990's, and with nanowires and nanocavities [4] [10] in 2000's. The surface topography has a major role to increase the nucleation sites number and that the sites must satisfy particular geometric and energetic minimal conditions to become active. Altering surface topography mainly acts on the nucleation sites density and consequently on interaction mechanisms, such as bubble coalescence, which should improve the heat transfer coefficients, but may promote the Critical Heat Flux (CHF) condition to occur at lower superheats. Several works have shown that even nanometric features influence nucleation by using highly smooth surfaces [11]. Differences of roughness on highly polished bronze surfaces down to few nanometers in amplitude modify the superheat needed to activate the boiling phenomenon [7]. On the other side, single cavities with a size of the order of 10 microns on silicon wafers trigger the boiling [12] [13], and their shape is also of primary importance [14] [15] for the nucleation.

The wettability quantifies the extent at which the surface keeps wetted by the liquid, and since 1980's many experimental studies have reported that surface wettability is an important factor affecting the boiling heat transfer [7] [16]. Measurements on wettability effects on bubble nucleation [7] [16], boiling heat transfer coefficients (HTC) [5] [6] [16] [17], critical heat flux [5] [17] [18] [19], bubble departure diameter [6] [20], and influence of wettability on the onset of boiling [2] have been carried out. It has been shown that the wettability affects the pool boiling triggering the nucleate boiling to start at lower superheat. At low heat fluxes higher contact angles (hydrophobic/superhydrophobic conditions) promote the activation of the nucleation sites [21] and the regular departure of larger bubbles, which are more likely to coalesce and buoyancy This mechanisms is consequently associated to an improvement of the heat transfer coefficient [22]. Obviously, the wettability affects the bubble dynamics (bubble growth, bubble departure diameter and bubble departure frequency). Bourdon et al. [16] performed experiments of incipient pool boiling of degassed water on glass substrates using surfaces with sub-nanometer roughness and different wettability. The reduction of the wettability induced a reduction of the onset of boiling: bubbles appear on the surface with lower superheat $(3.5 \mathrm{~K})$ on a hydrophobic surface with 
respect to a hydrophilic one (22K). As the heat flux increases and regime of slugs and columns occurs, large bubbles will actually be less beneficial because decreasing the heat transfer coefficient and promoting the establishment of a Critical Heat Flux condition. Hydrophobic surfaces lead to a fast formation of large vapor blankets thus CHF is reached at lower superheat. Betz et al. [23] suggest that dissimilar wettability conditions are required for the different heat transfer regimes. The use of biphilic surfaces, i.e. juxtaposition of hydrophilic and hydrophobic regions, is proposed. They show experimentally an overall better performance of these surfaces in pool boiling, when compared to those with spatially uniform wettability, both in terms of critical heat flux and heat transfer coefficient. The heat transfer coefficients measured on the so-called superbiphilic surfaces are up to three times higher than on state-of-the-art nanostructured surfaces with uniform wettability. Rioboo et al. [8] investigated a similar phenomenon for the flow boiling few years before, explaining the phenomenon with the presence of dissolved air nanobubbles on the superhydrophobic/hydrophilic borders. Takata et al. [24] studied experimentally pool boiling from a copper with the super-waterrepellent (SWR) coating of checkered and spotted patterns and $\mathrm{TiO}_{2}$-coated surface with polytetrafluoroethylene (PTFE) spotted patterns. Bubble nucleation occurs from the hydrophobic domain (SWR and PTFE) at very low superheating. In lower heat flux, bubbles with uniform size are generated on the SWR or PTFE domain of the heat transfer surface, and these bubbles depart from the heat transfer surface when the contact line reaches the boundary of SWR or PTFE domain. In case of subcooled condition, the nucleation begins at surface temperature below saturation temperature and bubbles generated and attached on the SWR surface merged into each other and finally formed film boiling. During this process there were no bubbles detaching from the surface and the observed transition to film boiling is completely different from that usually observed. Moreover, decreasing surface temperature starting from the film boiling regime, vapor film did not disappear, even if the surface temperature fell below the fluid saturation temperature.

In this work, pool boiling experiments were performed with degassed water on stainless steel substrates with different surface topographies and wettabilities. Boiling curves and visual observations of the boiling process have been performed. The superheat needed to have the onset of boiling on hydrophilic, hydrophobic and superhydrophobic surfaces (SHS) has been measured, and the influence of surface roughness and wettability has been quantified.

\section{Experimental}

\subsection{Pool boiling experimental setup}

The pool boiling set up is described in [16] in details. The boiling chamber is made in aluminum and an internal heater $(80 \mathrm{~W})$ heats up the water. Moreover, two external heating tapes are placed on the walls of the chamber and are connected to a PID controller to balance thermal leakage in order to maintain the saturation temperature in the chamber. Two K-thermocouples are placed in the water and connected to a proportional-integral-derivative (PID) controller to check the water temperature. A pressure gauge is connected to the tank to provide the value of the pressure inside the chamber and a bellows is used to modify the internal pressure of the chamber in order to work at saturation conditions. A ceramic cartridge (Acim Jouanin H6.5X32X175) of 175 Watts is placed in a house made in copper to heat the surface and reach boiling on it; this heater is surrounded by Teflon. A heat flux meter with 3 embedded T-thermocouples (Captec, France) is placed between the copper housing of the heater and the tested surface to provide a direct measure of the heat flux to the tested surface. A spring is placed between the bottom of the copper housing and the bottom of the Teflon box to press the heater with a constant strength on the surface. All the thermocouples and the pressure gauge are connected to a computer using a data acquisition system (Agilent A34970A data acquisition/switch unit, USA). Figure 1 shows a scheme of the experimental setup. Furthermore, to acquire the boiling process, a high-speed camera HCC-1000 (VDS Vosskuhler) located in front of the chamber was used. The measurement accuracy of the K-thermocouples is $0.5 \mathrm{~K}$, and the accuracy of the pressure gauge is $0.1 \% \mathrm{FS}$, equivalentl to $5 \mathrm{hPa}$. 


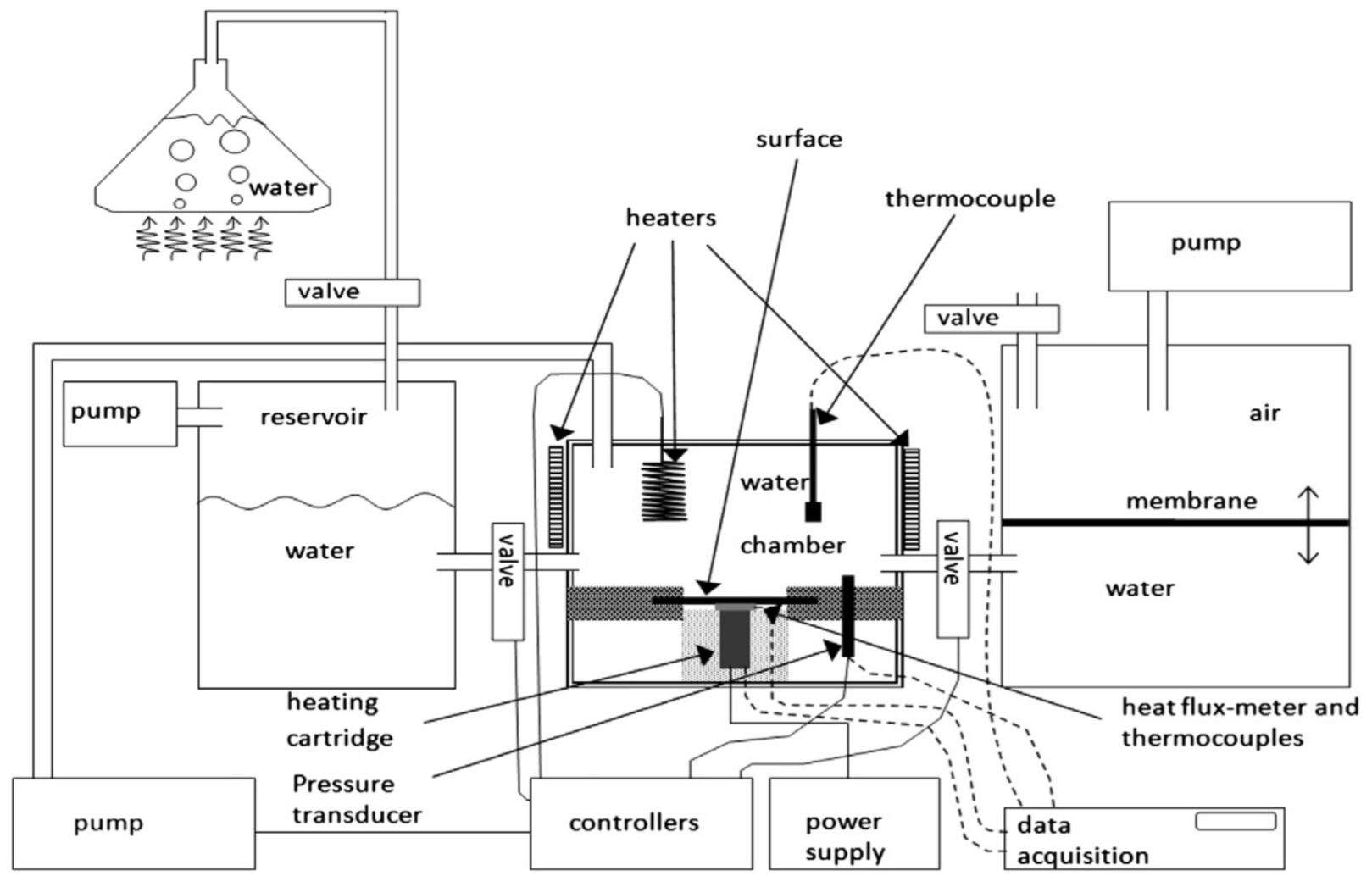

Figure 1 Experimental setup [16].

\subsection{Experimental procedure}

The chamber was vacuumed down to 30 mbar before adding water in order to remove air and adsorbed gases inside the tank. The chamber was then filled with a small quantity of degassed water, and vacuum was pulled once more in order to extract the residual air replacing it with water vapor. Finally the chamber was filled completely, and the internal heater was switched on in order to bring the water at its saturation temperature. When the water saturation temperature was reached, the internal heater was switched off and the cartridge heater was switched on to bring the copper housing at the saturation temperature of water. Having reached a null wall superheat (difference between the surface temperature $\mathrm{T}_{\text {wall }}$ and the water temperature at saturation $\mathrm{T}_{\text {sat }}$ ), the cartridge power was increased by a tiny amount $(1 \mathrm{~W})$ and temperature and flux measures were recorded. The boiling curve was built by slowly increasing step by step (max 5 volt each 5 minutes) the cartridge power, in order to work always in stationary conditions. i.e every power step was kept the necessary time to achieve a stable surface temperature (within $0.5 \mathrm{~K}$ ).

\subsection{Materials and preparation of the test surfaces}

With the goal to measure the influence of the surface topography and wettability on the boiling curve, and specifically the case of superhydrophobic surfaces, treated steel surfaces (stainless steel 304S15, $1 \mathrm{~mm}$ thickness) were used. It was decided to use stainless steel because it is a material widely used for industrial applications, very replicable and therefore interesting also from the practical point of view. Knowing that the surface durability of the superhydrophobic coatings is still an important issue [25], after different tests with different surface treatments, the superhydrophobic surfaces able to resist the thermal stress during at least two boiling curves have been finally produced using a coating prepared with a commercial product - Glaco Mirror Coat "Zero" (Soft 99 Co.). As reported in the supplementary information of [26], it is an alcohol-based suspension of silica nanoparticles functionalized by organic hydrophobizing agent.

Boiling experiments were carried out on 6 different types of surfaces:

- $\quad$ stainless steel as by purchase, hereinafter referred to as Raw;

- $\quad$ rough stainless steel, obtained by inserting the normal surface in a solution $6 \mathrm{M}$ of distilled water and hydrochloric acid for 15 minutes, then rinsed with distilled water and dried in air, hereinafter referred to as RoAc;

- $\quad$ rough stainless steel, obtained by grinding, with circular motion, a normal surface with sandpaper 500. A blasting process in order to obtain a more homogeneous roughness was not possible, due to the surface deformations. Using surfaces with greater thicknesses did not allow the necessary temperature homogeneity for the boiling tests. This surfaces is hereinafter referred to as RoSand;

- stainless steel as by purchase, coated with a commercial coating agent containing nanoparticles and organic reagent, hereinafter referred to as RawG_SHS; 
- rough stainless steel, based on RoAc, coated with a commercial coating agent containing nanoparticles and organic reagent, hereinafter referred to as RoAcG_SHS;

- $\quad$ rough stainless steel, based on RoSand, coated with a commercial coating agent containing nanoparticles and organic reagent, hereinafter referred to as RoSandG_SHS.

The following procedure has been used for cleaning the surfaces: surfaces have been immersed in water at $80^{\circ} \mathrm{C}$ for 30 minutes, then inserted in acetone at $60^{\circ} \mathrm{C}$, and finally in alcohol at $60^{\circ} \mathrm{C}$. All the procedure was made in an ultrasonic bath. To coat the surfaces with the Glaco Mirror the following procedure has been used:

- $\quad$ the surfaces were cleaned and dry in standard air under the hood for $24 \mathrm{~h}$;

- $\quad$ the surfaces were placed in a vertical position, then sprayed with the Glaco Mirror until a thin liquid film weted the entire surface, and finally left to dry in air under the hood for $24 \mathrm{~h}$.

\subsection{Surface characterization}

The roughness of the surfaces was measured using a confocal microscopy and interferometry (Sensofar PLu Neox, Spain). The results are presented in Table 1. The mean roughness is quantified by the 3D parameters Sa and Sq. Sa expresses the average of the absolute values of the profile heights $\mathrm{Z}(\mathrm{x}, \mathrm{y})$ in the measured area. Sq expresses the root mean squared of $\mathrm{Z}(\mathrm{x}, \mathrm{y})$ in the measured area. Since the key parameter for the incipient boiling is not only the mean roughness, but either the peak-to-valley depth or a parameter able to properly quantify the size of cavities present on the surface [7], also Sv and Sz parameters are evaluated. Sv expresses the maximum value valley depth on the surface in the measured area. Sz expresses the sum of the maximum value of peak height and the maximum value of valley depth on the surface within the measured area.

\section{Table 1}

Roughness parameters for the substrates.

\begin{tabular}{|c|rrrrrr|}
\hline & Raw $[\boldsymbol{\mu m}]$ & RawG_SHS $[\boldsymbol{\mu m}]$ & RoAc $[\boldsymbol{\mu m}]$ & RoAcG_SHS $[\boldsymbol{\mu m}]$ & RoSand $[\boldsymbol{\mu m}]$ & RoSandG_SHS $[\boldsymbol{\mu m}]$ \\
\hline Sa & $\mathbf{1 , 6 7 2}$ & $\mathbf{2 , 0 4 4}$ & $\mathbf{8 , 1 0 8}$ & $\mathbf{9 , 9 8 3}$ & $\mathbf{2 , 5 3 7}$ & $\mathbf{2 , 8 4 9}$ \\
St Dev Sa & 0,016 & 0,111 & 0,155 & 0,342 & 0,103 & 0,078 \\
Sq & $\mathbf{2 , 0 9 1}$ & $\mathbf{2 , 6 0 8}$ & $\mathbf{1 2 , 9 0 9}$ & $\mathbf{7 7 , 8 3 1}$ & $\mathbf{3 , 5 1 6}$ & $\mathbf{4 , 0 2 7}$ \\
St Dev Sq & 0,021 & 0,102 & 0,282 & 18,895 & 0,163 & 0,151 \\
Sv & $\mathbf{1 0 , 9 6 0}$ & $\mathbf{1 4 , 0 5 8}$ & $\mathbf{1 4 0 , 6 8 1}$ & $\mathbf{2 0 2 , 6 0 6}$ & $\mathbf{8 5 , 4 9 4}$ & $\mathbf{9 4 , 2 1 5}$ \\
St Dev Sv & 1,422 & 0,938 & 2,141 & 2,848 & 2,269 & 2,588 \\
Sz & $\mathbf{2 2 , 4 5 6}$ & $\mathbf{2 8 , 1 9 8}$ & $\mathbf{2 1 5 , 1 8 8}$ & $\mathbf{2 5 3 , 8 1 0}$ & $\mathbf{1 0 5 , 4 3 7}$ & $\mathbf{1 2 6 , 8 9 7}$ \\
St Dev Sz & 1,362 & 1,275 & 2,787 & 2,968 & 1,952 & 2,458 \\
\hline
\end{tabular}

The Glaco Mirror layer, added to the stainless steel substrate to modify its wettability, affects also its mean roughness, as shown in Table 1. Surfaces RoSand and RoSandG_SHS may be not completely homogeneous, since at the microscope it is possible to detect scratches due to the sandpaper and the procedure to prepare them. See supplementary material A for more information.

To define the surface wettability, advancing and receding contact angles, $\theta_{\mathrm{A}}$ and $\theta_{\mathrm{R}}$, were classically measured by expanding and contracting sessile water drops on the horizontal surface (sessile drop method) [27]. $\theta_{\mathrm{A}}$ and $\theta_{\mathrm{R}}$ measurements are shown in Table 2. Typical drop volume of the analyzed drops was $\sim 5 \mu 1$. To measure the contact angle, the drop profile in the vicinity of the contact line was extracted and fitted with a circle; the contact angle was computed from the slope of the circle at the surface [28] [29]. It is here necessary to specify that the contact angles, and in particular the superhydrophobicity of the surfaces, were maintained during the boiling test, as evidenced by the work of Yu et al. [30]. They noticed that superhydrophobic surfaces maintain their wettability when the surface temperature is greater than of the water temperature.

Table 2

Average $\theta_{\mathrm{A}}$ and $\theta_{\mathrm{R}}$ for the substrates. The standard deviations are lower than $\pm 3^{\circ}$.

\begin{tabular}{|l|rrrrrr|}
\hline & Raw & RawG_SHS & RoAc & RoAcG_SHS & RoSand & RoSandG_SHS \\
\hline $\boldsymbol{\theta}_{\mathbf{A}}\left[{ }^{\circ}{ }^{\circ}\right]$ & 91 & 151 & 29 & 149 & 72 & 153 \\
$\boldsymbol{\theta}_{\mathbf{R}}\left[{ }^{\circ}\right]$ & 53 & 150 & $<20$ & 149 & 31 & 152 \\
\hline
\end{tabular}

The chemical composition of each surface was analyzed using a SEM-EDX (Bruker Nano GmbH, K-series). The raw data of the normalized percentage composition by weight of each element are shown in Table 3.

Table 3

Normalized percentage composition by weight of each element [norm. wt.\%] for each surface type (average values).

\begin{tabular}{|c|rrrrrr|}
\hline Element & Raw & RawG_SHS & RoAc & RoAcG_SHS & RoSand & RoSandG_SHS \\
\hline iron & 67,54 & 60,05 & 65,37 & 60,89 & 65,16 & 61,19 \\
chrome & 15,21 & 13,74 & 16,12 & 15,35 & 14,73 & 14,14 \\
\hline
\end{tabular}




\begin{tabular}{|c|rrrrrr|} 
nickel & 7,40 & 6,15 & 6,88 & 6,47 & 7,09 & 6,65 \\
carbone & 7,96 & 5,66 & 7,15 & 6,02 & 8,97 & 8,38 \\
manganese & 1,89 & 1,67 & 2,15 & 1,84 & 1,93 & 1,81 \\
oxygene & 0,00 & 8,34 & 1,87 & 6,68 & 2,12 & 5,14 \\
silicium & 0,00 & 4,40 & 0,00 & 2,75 & 0,00 & 2,71 \\
\hline Sum: & 100,00 & 100,00 & 100,00 & 100,00 & 100,00 & 100,00 \\
\hline
\end{tabular}

After every boiling test, measures of roughness, contact angle and chemical composition analysis were again performed. Results do not show any discrepancy with the analysis performed before the boiling tests.

\section{Results and discussion}

In Figure 2 boiling curves with the standard deviation of data and the onset of nucleate boiling (ONB) are presented for each surface. ONB is here determined by a visualization of the incipient boiling. For the raw stainless steel (Raw) ONB appears at a superheat of $12^{\circ} \mathrm{C}$. In the case of rough steel surfaces (RoAc and RoSand) the ONB appears at slightly lower superheat (between 7 and $10^{\circ} \mathrm{C}$ ). At the boiling onset only few small bubbles appear on Raw, RoAc and RoSand surfaces (Figure 3) and even when the superheat reaches $30^{\circ} \mathrm{C}$ a small number of tiny bubbles is present on the surfaces. On the Raw surface, bubbles are rapidly upward elongated and detach from the surface (see Video in supplementary material B), while on RoAc and RoSand surfaces, the bubbles are more spherical, and detach rapidly from the surface, facilitating the rewetting of the boiling surface. The vapour bubbles on the RoAc surface have a lower diameter with respect to the bubbles on the RoSand surface, which has higher contact angles. Hence, keeping the same surface roughness, bubble departure diameter decreases with the decrease of the contact angle, and a lower superheat is required for bubble nucleation on the rough surfaces with respect to the raw surface.

In the case of superhydrophobic surfaces (RawG_SHS, RoAcG_SHS and RoSandG_SHS), the ONB appears at superheat very close to zero (i.e. at the first step of increasing power), and sometimes first small bubbles are formed even when the surface has a temperature lower than the water saturation temperature. Therefore it seems that the initial gas film is sometimes formed not by the vapour, but by the air initially trapped between the roughness peaks of the superhydrophobic surfaces, even if the water was properly degassed. The presence of such air bubble entrapped in the surface cavities can explain also other literature results [24] [26]. This outcome confirms also what Carey reported in his book [31] for rough surfaces, even without an explicit reference to experimental results: "If the liquid does not wet the heated surface, vapor and/or air will be trapped in virtually every cavity on the surface... Vaporization is initiated immediately when the surface temperature begins to exceed saturation ... and the vapor produced at one location will displace liquid adjacent to the surface and spread laterally to form a vapor blanket on the surface. Thus, once boiling is initiated, the boiling process immediately enters the film boiling regime".

On superhydrophobic surfaces, as soon as the flux increases, in few seconds the bubbles coalesce and form one single bubble, like a steam blanket, which covers most of the heated surface. When a bubble is detached from a superhydrophobic surface another bubble immediately forms, i.e. a thin vapor layer is present in a constant mode after the ONB. Therefore the vapour film formed on the SHS is stable, as also shown for a different experiment and application by Vakarelski et al. [26]. When the superheat is about $30^{\circ}$ the departure frequency is reaching a value of approximately $1 \mathrm{~Hz}$ and intermittent rewetting occurs. Such typical bubble dynamic behavior on SHS is shown in Figure 4 and in the supplementary material C.
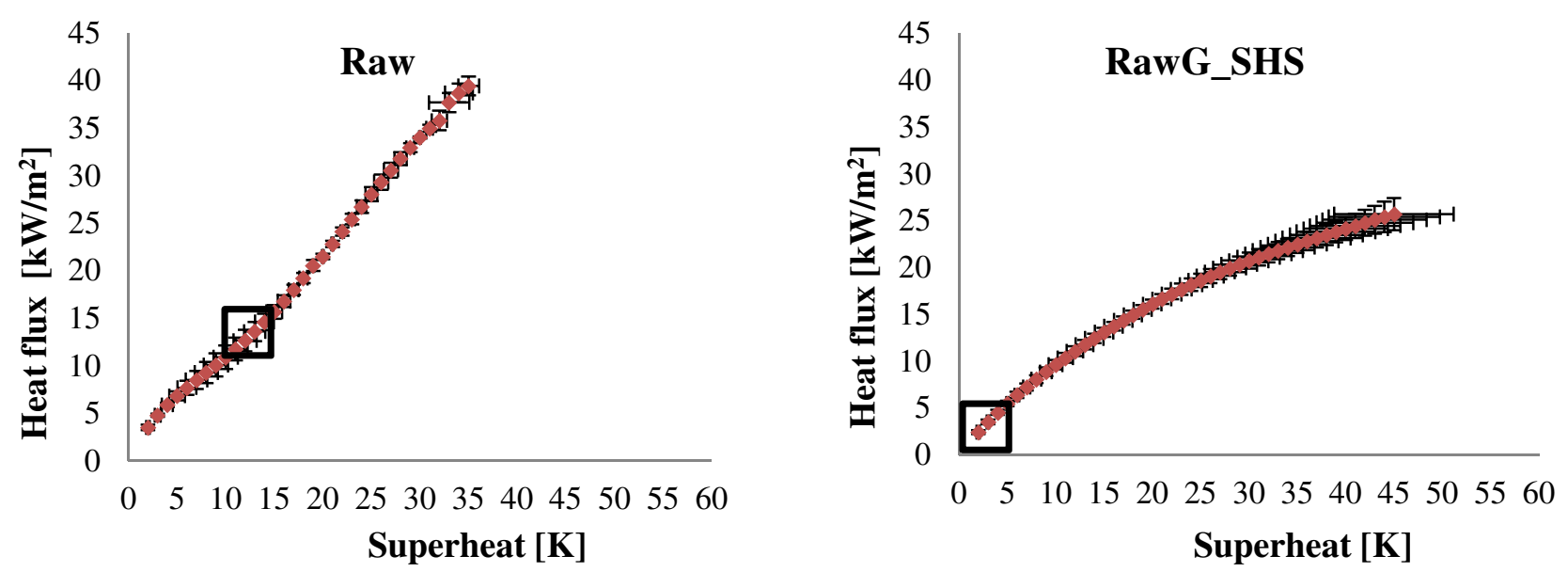

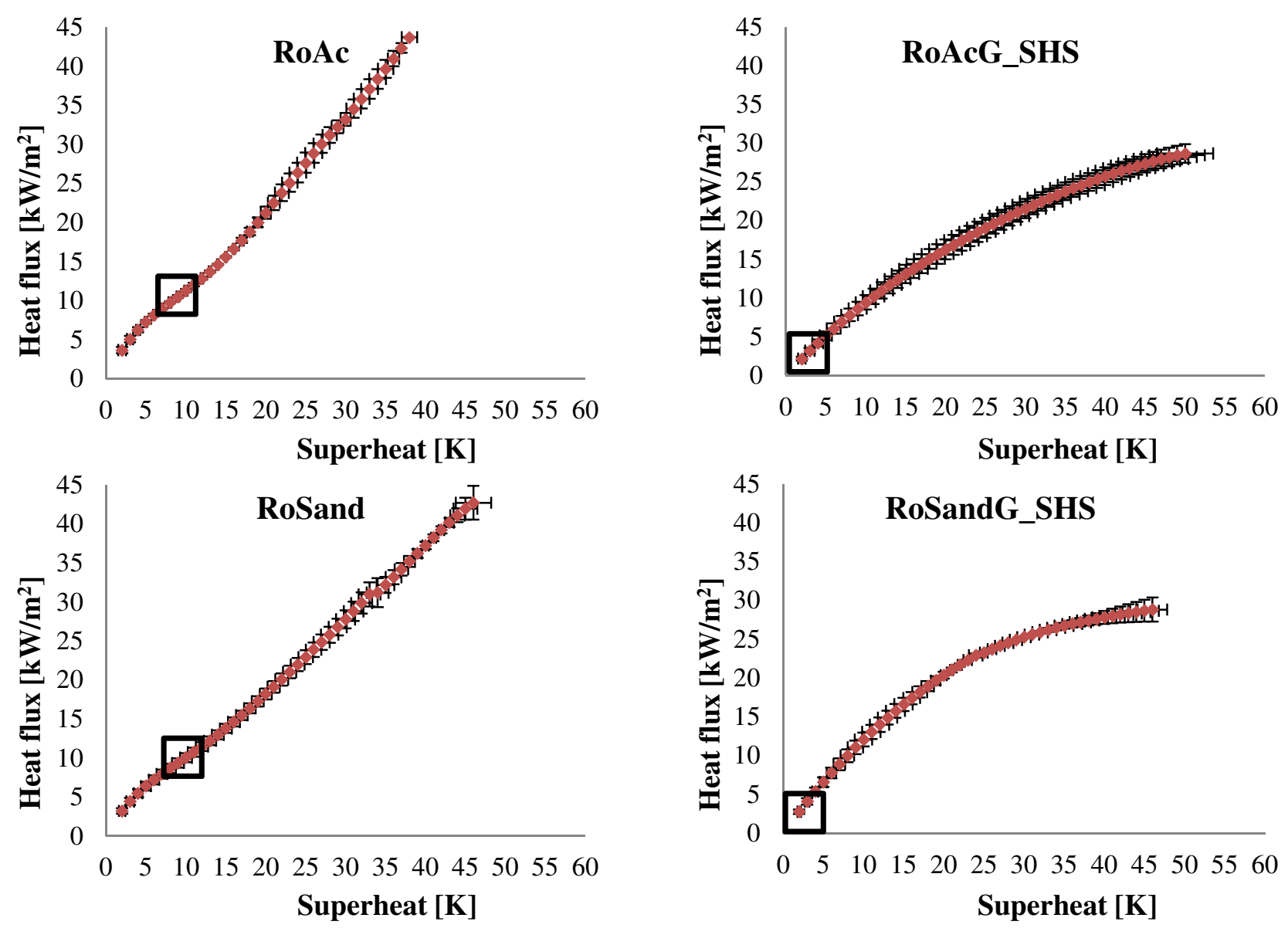

206

207 208
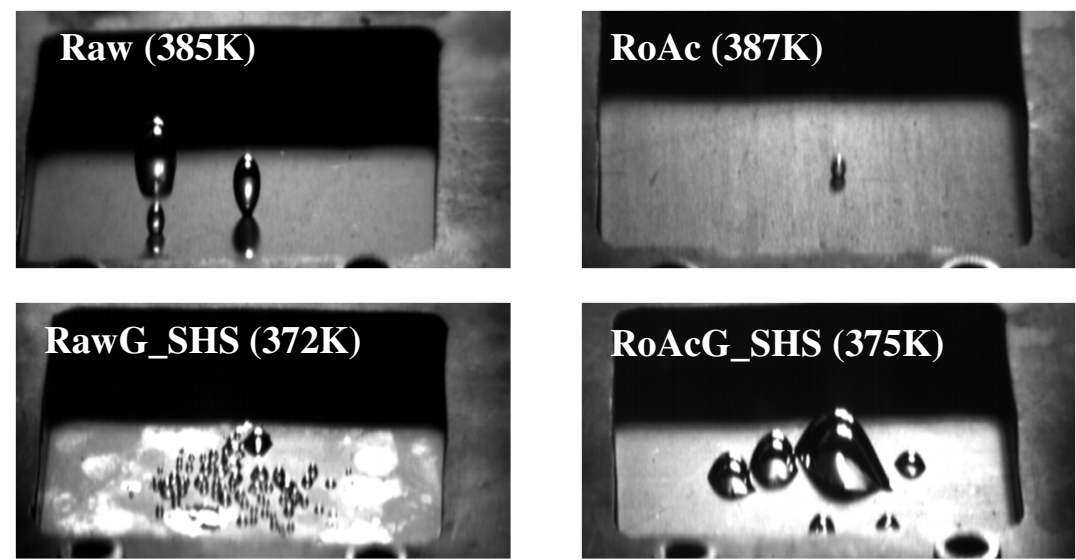
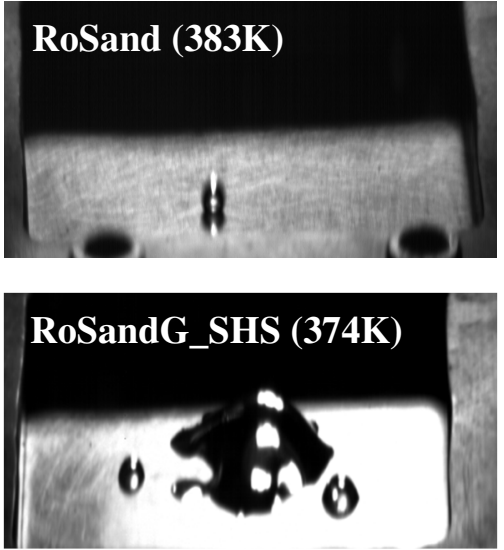

Figure 3 Incipient boiling. Between brackets the surface temperature to which the image refers. The error on the temperature is about \pm 0.5 . Pressure $=P_{\text {sat. }}$.
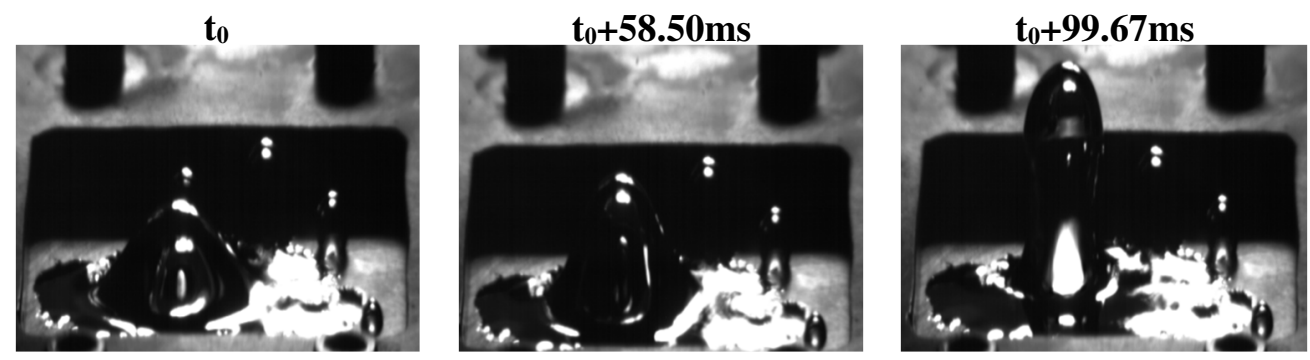

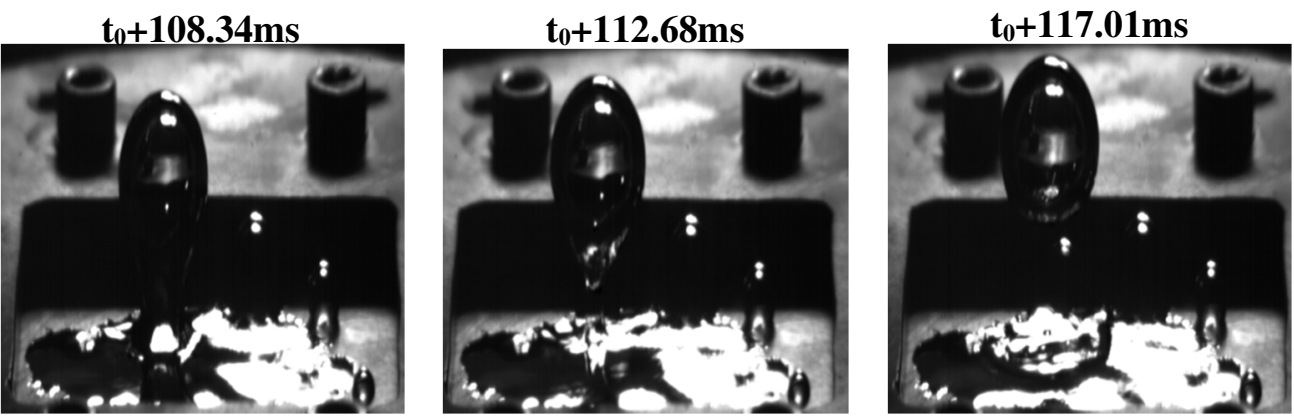

Figure 4 Bubble dynamics on the surface RoSandG_SHS. The time (with respect to the time of the first image) is reported above the image.

A significant change in the shape of the boiling curve is visible in Figure 5. The slope of the boiling curves for Raw, RoAc and RoSand cases suggests a standard boiling behavior (solid symbols), and a higher heat removal with respect to the superhydrophobic surface cases (open symbols). From Figure 5a the boiling curves for the SH surfaces RawG_SHS, RoAcG_SHS and RoSandG_SHS are almost constant around a heat flux equal to $25 \mathrm{~kW} / \mathrm{m}^{2}$, i.e. a further increase of the heat flux is bringing the surfaces to very high values of temperature. From Figure 5a it is also possible to see that the curve referred to surface Raw is practically superimposed to RoAc boiling curve. The surface RoAc has a higher roughness and a higher wettability respect surface Raw, i.e. $\theta_{\mathrm{A}}$ of $29^{\circ}$ instead of $91^{\circ}$ respectively. It follows that, when the surfaces have a low contact angle, the surface roughness is controlling the boiling process. In Figure 5b the heat transfer coefficients are shown: it is possible to notice a marked difference of the HTC between superhydrophobic surfaces and their corresponding reference case, confirming the fact that superhydrophobic surfaces do not follow the typical trend of known boiling curves. SHS surfaces show a peculiar behavior reaching a sort of early Leidenfrost regime, i.e. a lower HTC just after the ONB.
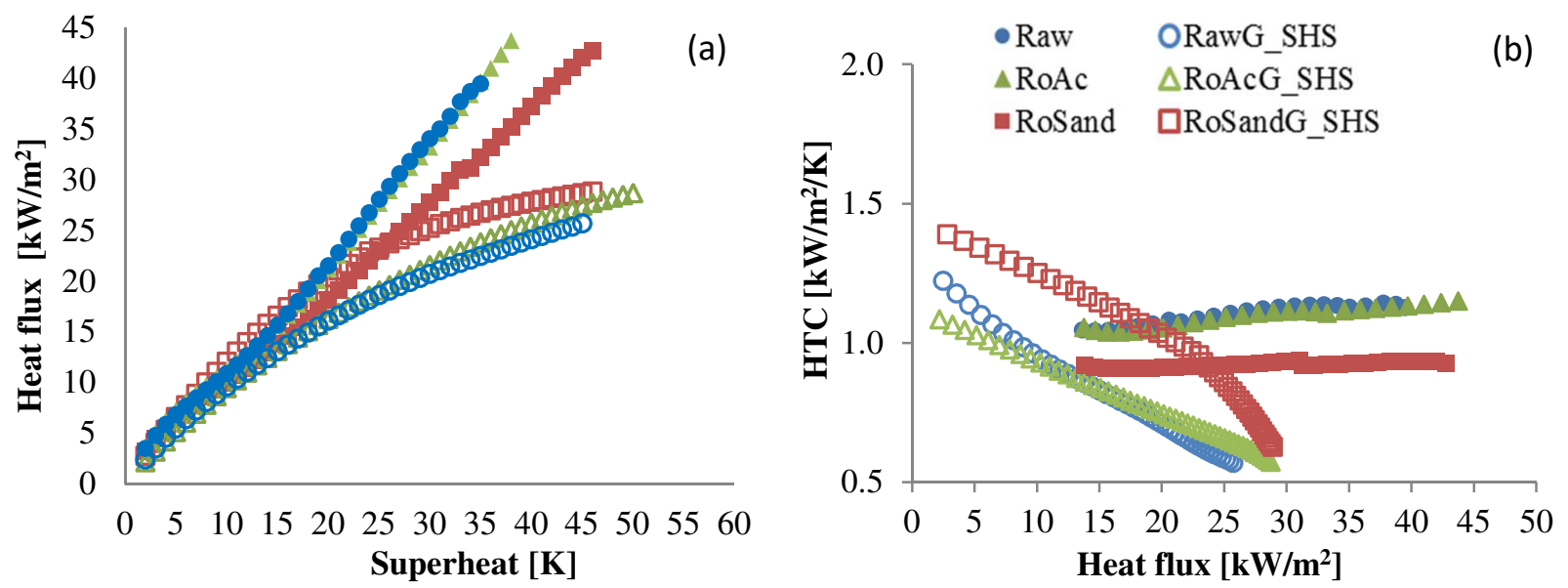

\section{Hydrophilic}

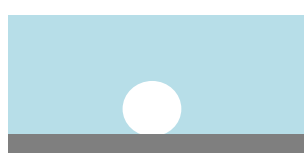

SHS

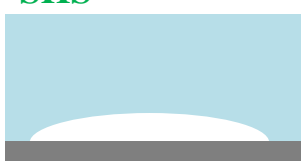

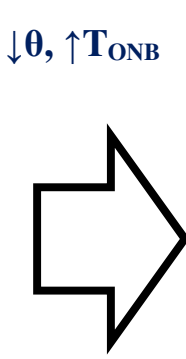

$\uparrow \boldsymbol{\theta}, \downarrow \mathbf{T}_{\mathrm{ONB}}$

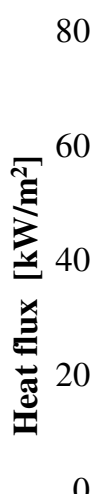

0

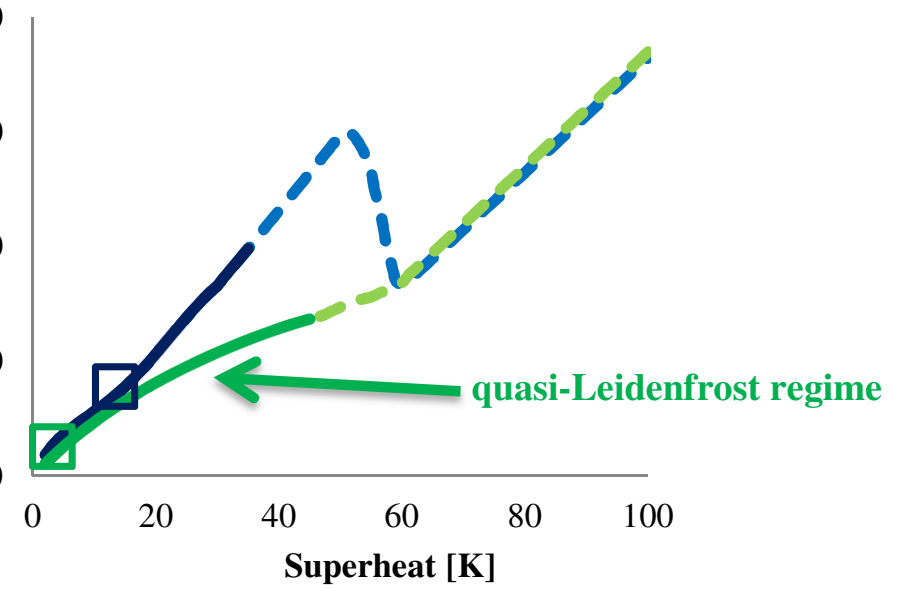


Figure 6 Schematic representation of the bubble formation on hydrophilic and superhydrophobic surfaces with the corresponding boiling curve. Continuous lines represent the experimental curves found for hydrophilic (blue line) and SHS (green line) cases. Dashed lines represent the respective boiling curves hypothesized as a result of the experimental data obtained; the Captec flux sensor did not allow higher temperatures. In the box is highlighted the ONB for the respective cases.

\section{Conclusions}

Pool boiling experiments were carried out with degassed water on stainless steel substrates with different surface topography and wettability. Boiling curves have been measured together with visual high-speed observations of the boiling process. The onset temperature of nucleate boiling (ONB) has been measured and the influence of surface roughness and wettability has been quantified for different surfaces, with the aim to better understand the effect of superhydrophobicity on pool boiling. The original finding is that the boiling curve shape is rather different between hydrophilic and superhydrophobic case, keeping the same surface roughness, as schematically depicted in Figure 6. Superhydrophobic surfaces show a peculiar behavior similar to an early "quasi-Leidenfrost" regime for low superheat. The ONB appears after less than $5 \mathrm{~K}$ of superheat on superhydrophobic surfaces, while, for the hydrophilic cases the ONB appears at more than $7 \mathrm{~K}$ of superheat, which is a standard value using water on steel for a mean surface roughness above $2 \mu \mathrm{m}$ [32]. Bubble nucleation on SHS builds up a large and stable vapour film with a very long departure time of the order of one minute. Only when the superheat is about $15 / 20 \mathrm{~K}$ the departure time starts decreasing and it get down to the more standard value of one second. Regardless of the surface roughness and topology, the boiling curves obtained with the superhydrophobic surfaces (RawG_SHS, RoAcG_SHS and RoSandG_SHS) are very similar. This means that the wettability has a predominant effect with respect to the roughness, when the contact angle exceeds the typical value for superhydrophobic surfaces (receding contact angle $\theta_{\text {rec }}$ $>135^{\circ}$ ).

\section{Acknowledgments}

This work is supported by the Italian Ministry of University and Research (PRIN 2009), by the Belgian National Fund for Scientific Research (FRS-FNRS) for a doctoral grant FRIA and, partially, by the European Cooperation in Science and Technology - MPNS COST Action MP1106 "Smart and green interfaces - from single bubbles and drops to industrial, environmental and biomedical applications" led by Prof. T. Karapantsios. The authors would like also to thank Mirko Carminati for the help in experiments and data analysis.

\section{References}

[1] K. Ferjancic, I. Golobic, Surface effects on pool boiling CHF, Experimental Thermal and Fluid Science 25 (2002) $565-571$.

[2] I.L. Pioro, W. Rohsenow, S.S. Doerffer, Nucleate pool-boiling heat transfer. I: review of parametric effects of boiling surface, International Journal of Heat and Mass Transfer 47 (2004) 5033-5044.

[3] H.T. Phan, R. Bertossi, N. Caney, P. Marty, S. Colasson, A model to predict the effect of surface wettability on critical heat flux, International Communication of Heat and Mass Transfer 39 (2012) 1500-1504.

[4] Z. Yao, Y.W. Lu, S.G. Kandlikar, Effects of nanowire height on pool boiling performance of water on silicon chips, International Journal of Thermal Sciences 50 (2011) 2084-2090.

[5] H.J. Jo, H.S. Ahn, S.H. Kang, M.H. Kim, A study of nucleate boiling heat transfer on ydrophilic, hydrophobic and heterogeneous wetting surfaces, International Journal of Heat and Mass Transfer 54 (2011) 5643-5652.

[6] H.T. Phan, N. Caney, P. Marty, S. Colasson, J. Gavillet, Surface wettability control by nanocoating: the effects on pool boiling heat transfer and nucleation mechanism, International Journal of Heat and Mass Transfer 52 (2009) 5459-5471.

[7] B. Bourdon, R. Rioboo, M. Marengo, E. Gosselin, J. De Coninck, Influence of the wettability on the boiling onset, Langmuir 28 (2012) 1618-1624.

[8] R. Rioboo, M. Marengo, S. Dall'Olio, M. Voué, J. De Coninck, An innovative method to control the incipient flow boiling through grafted surfaces with chemical patterns, Langmuir 25 (2009) 6005-6009.

[9] K.H. Chu, R. Enright, E.N. Wang, Structured surfaces for enhanced pool boiling heat transfer, Applied Physics Letters 100 (2012) 241603.1-3.

[10] L.N. Dong, X.J. Quan, P. Cheng, An experimental investigation of enhanced pool boiling heat transfer from surfaces with micro/nano-structures, International Journal of Heat and Mass Transfer 71 (2014) 189-196.

[11] Y. Nam, J. Wu, G. Warrier, Y.S. Ju, Experimental and numerical study of single bubble dynamics on a hydrophobic surface, Journal of Heat Transfer 31 (2009) 121004.1-7.

[12] C. Hutter, D.B.R. Kenning, K. Sefiane, T.G. Karayiannis, H. Lin, G. Cummins, A.J. Walton, Experimental pool boiling investigations of FC-72 on silicon with artificial cavities and integrated temperature microsensors, Experimental Thermal and 
Fluid Science, 34 (2010) 422-433.

[13] C. Hutter, K. Sefiane, T.G. Karayiannis, A.J. Walton, R.A. Nelson, D.B.R. Kenning, Nucleation site interaction between artificial cavities during nucleate pool boilingon silicon with integrated micro-heater and temperature micro-sensors, International Journal of Heat and Mass Transfer 55 (2012) 2769-2778.

[14] H. Honda, J.J. Wei, Enhanced boiling heat transfer from electronic components by use of surface microstructures, Experimental Thermal and Fluid Science 28 (2004) 159-169.

[15] L.N. Dong, X. Quan, P. Cheng, An analysis of surface-microstructures effects on heterogeneous nucleation in pool boiling, International Journal of Heat and Mass Transfer 55 (2012) 4376-4384.

[16] B. Bourdon, P. Di Marco, R. Rioboo, M. Marengo, J. De Coninck, Enhancing the onset of pool boiling by wettability modification on nanometrically smooth surfaces, International Communications in Heat and Mass Transfer 45 (2013) 11-15.

[17] A.R. Betz, J. Xu, H. Qiu, D. Attinger, Do surfaces with mixed hydrophilic and hydrophobic areas enhance pool boiling?, Applied Physics Letters 97 (2010) 141909.1-3.

[18] Y. Takata, S. Hidaka, J.M. Cao, T. Nakamura, H. Yamamoto, M. Masuda, T. Ito, Effect of surface wettability on boiling and evaporation, Energy 30 (2005) 209-220.

[19] S.J. Kim, I.C. Bang, J. Buongiorno, L.W. Hu, Surface wettability change during pool boiling of nanofluids and its effect on critical heat flux, International Journal of Heat and Mass Transfer 50 (2007) 4105-4116.

[20] H.T. Phan, N. Caney, P. Marty, S. Colasson, J. Gavillet, A model to predict the effect of contact angle on the bubble departure diameter during heterogeneous boiling, International Communications in Heat and Mass Transfer 37 (2010) 964-969.

[21] C.H. Wang, V.K. Dhir, Effect of surface wettability on active nucleation sited density during pool boiling of water on a vertical surface, Journal of Heat Transfer 115 (3) (1993) 659-669.

[22] M.G. Cooper, The microlayer and bubble growth in nucleate pool boiling, International Journal of Heat and Mass Transfer 12 (1969) 915-933.

[23] A.R. Betz, J. Jenkins, C.J. Kim, D. Attinger, Boiling heat transfer on superhydrophilic, superhydrophobic, and superbiphilic surfaces, International Journal of Heat and Mass Transfer 57 (2013) 733-741.

[24] Y. Takata, S. Hidaka, M. Kohno, Effect of surface wettability on pool boiling: enhancement by hydrophobic coating, International Journal of Air-Conditioning and Refrigeration 20 (1) (2012) 1150003.1-9.

[25] I. Malavasi, I. Bernagozzi, C. Antonini, M. Marengo, Towards a standard protocol for assessing durability of superhydrophobic surfaces, Surface Innovations Journal DOI: 10.1680/si.14.00001.

[26] I.U. Vakarelski, N.A. Patankar, J.O. Marston, D.Y.C. Chan, S.T. Thoroddsen, Stabilization of Leidenfrost vapour layer by textured superhydrophobic surfaces, Nature 489 (2012) 274-277.

[27] J. Drelich, Guidelines to measurements of reproducible contact angles using a sessile-drop technique, Surface Innovations 1 (2013) 248-254.

[28] C. Antonini, A. Amirfazli, M. Marengo, Drop impact and wettability: from hydrophilic to superhydrophobic surfaces, Physics of Fluids 24 (2012) 102104.1-13.

[29] C. Antonini, F. Villa, I. Bernagozzi, A. Amirfazli, M. Marengo, Drop rebound after impact: the role of the receding contact angle, Langmuir 29 (2013) 16045-16050.

[30] Z.J. Yu, J. Yang, F. Wan, Q. Ge, L.L. Yang, Z.L. Ding, D.Q. Yang, E. Sacherc, T.T. Isimjand, How to repel hot water from a superhydrophobic surface?, Journal of Materials Chemistry A: Materials for Energy and Sustainability 2 (2014) 10639-10646.

[31] V.P. Carey, Liquid vapor phase change phenomena: an introduction to the thermophysics of vaporization and condensation processes in heat transfer equipment, second ed., Taylor \& Francis, 2007, pp. 307.

[32] B.J. Jones, J.P. McHale, S. Garimella, The influence of surface roughness on nucleate pool boiling heat transfer, Journal of Heat Transfer 131 (12) (2009) 121009.1-14. 